\title{
Production planning in a construction company as an element of Gartner enterprise architecture
}

\author{
S S Valeev ${ }^{1,2}$, N V Kondratyeva ${ }^{2}$, R R Karimov ${ }^{1}$, M A Verkhoturov ${ }^{1}$, \\ T V Islamgulov ${ }^{1}$ and $L$ I Shekhtman ${ }^{1}$ \\ ${ }^{1}$ Ufa State Aviation Technical University, K. Marx Str. 12, Ufa, 450008, Russia \\ ${ }^{2}$ Sochi State University, Politekhnicheskaya Str. 7, Sochi, 354008, Russia
}

vss2000@mail.ru

\begin{abstract}
The problem of production planning as an element of Gartner enterprise architecture in a distributed organizational and technical system is considered on the example of a construction company of medium and large scale. Mathematical models for planning organizational and technical resources in a distributed system as elements of patterns are discussed. In the development of the information and application layers, field-proven solutions can be used as patterns. The approach to the organization of business processes of a construction company that allows solving the problem of optimal scheduling of construction works while simultaneously executing a number of projects by the company and, thus, increasing the management efficiency of a geographically distributed enterprise is considered. The enterprise architecture as a unified information environment for managers of a construction company, site managers, supply engineers, designers, and estimates is discussed.
\end{abstract}

\section{Introduction}

Construction companies attribute to the class of distributed large-scale systems, characterized by a multi-level management system and a geographically distributed structure. Since they simultaneously implement business processes at several geographically remote sites, in order to solve their production tasks, various subcontractors are involved in production and supplies.

To improve the efficiency of the construction company, various approaches are used, based on a phased transition from traditional data processing to a digital company within the framework of the Industry 4.0 concepts $[1,2]$. One such approach is the development of enterprise architecture. Enterprise architecture includes basic elements [3], patterns [4], business process models [5] and models of interaction with the external environment [6].

Construction business is associated with the expenditure of significant amounts of labor, material and financial resources on the facilities being built in parallel. At the planning stage of construction production, it is necessary effectively distribute various types of resources between construction facilities in order to increase the efficiency of the entire enterprise [7, 8]. Thus, in the face of market competition and a difficult financial situation, the company needs operational planning methods [9].

The task of planning and managing geographically distributed production divided into two levels the level of the local construction site and the level of the parent company [10]. 
Considering that the process of designing a work plan based on a mathematical model for optimizing time costs and optimal distribution of tasks, we will further consider these tasks as the main elements of the design of the architecture of a construction company.

The article discusses the formulation of the problem associated with the development of the architecture of a construction company as a distributed production system. To improve the efficiency of developing an information system, it is proposed to use an architectural approach in its development. As a basic architecture, it is proposed to use an enterprise architecture based on the Gartner architecture [3]. This enterprise architecture includes basic elements, patterns, business process models and models of interaction with the external environment. The following discusses the architectural features of developing an information system using this approach.

The mathematical formulation of the problem of optimizing business processes as a basic pattern is considered. This optimization model can serve as a basis for developing the architecture of a construction company.

The problem of developing a set of patterns for enterprise architects is considered on the example of patterns of basic business processes and the schedule of work of a construction company. A prototype of the architecture of a construction company based on the Gartner enterprise architecture is proposed.

\section{Statement of the problem}

Cost reduction in construction is carried out not only by rational space-planning solutions of buildings, the correct choice of building and finishing materials, lightening of construction, improvement of construction methods, but also by effective management of all activities of a construction company, prompt allocation of resources and optimization of the production plan [11, 12].

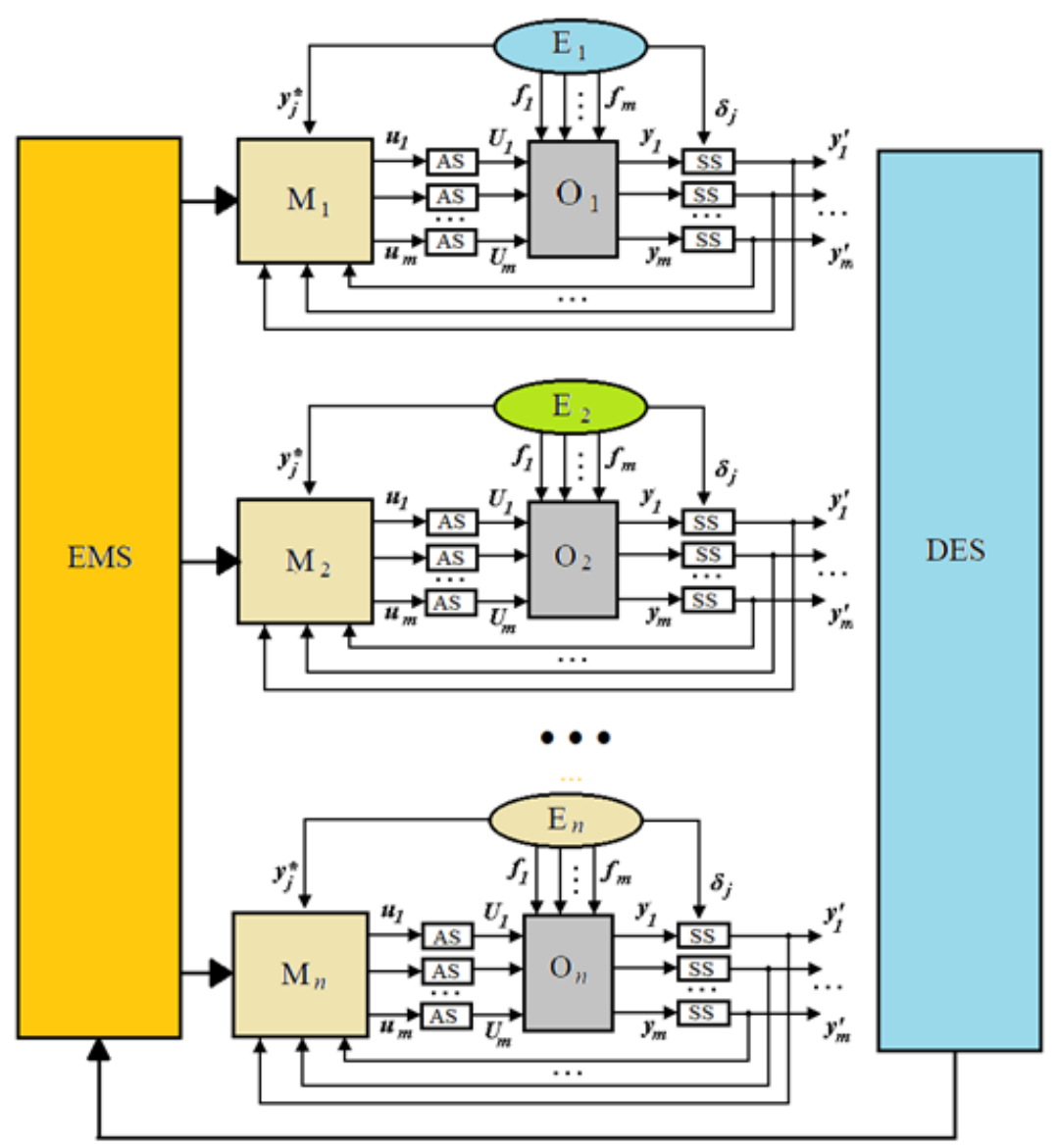

Figure 1. Scheme of information flows in Construction Company. 
Let us further consider the scheme of interaction between the parent enterprise and construction projects during production planning, presented in figure 1 . Here $M_{i}$ is the system of organizational and technical management of the $i$-th construction project, and then $E_{i}$ is environment for the $i$-th construction project. Elements $A S$ (actuator system) are subsystems for the implementation of control commands coming from a system $M_{i}$. Elements $S S$ (sensors system) are subsystems for collecting information about the state of business processes and resources. DES (distributed enterprise information system) is a system for collecting, storing and transmitting data for a construction company. EMS is an enterprise management system.

The task is to integrate all these subsystems based on enterprise architecture (see figure 2) [13, 14].

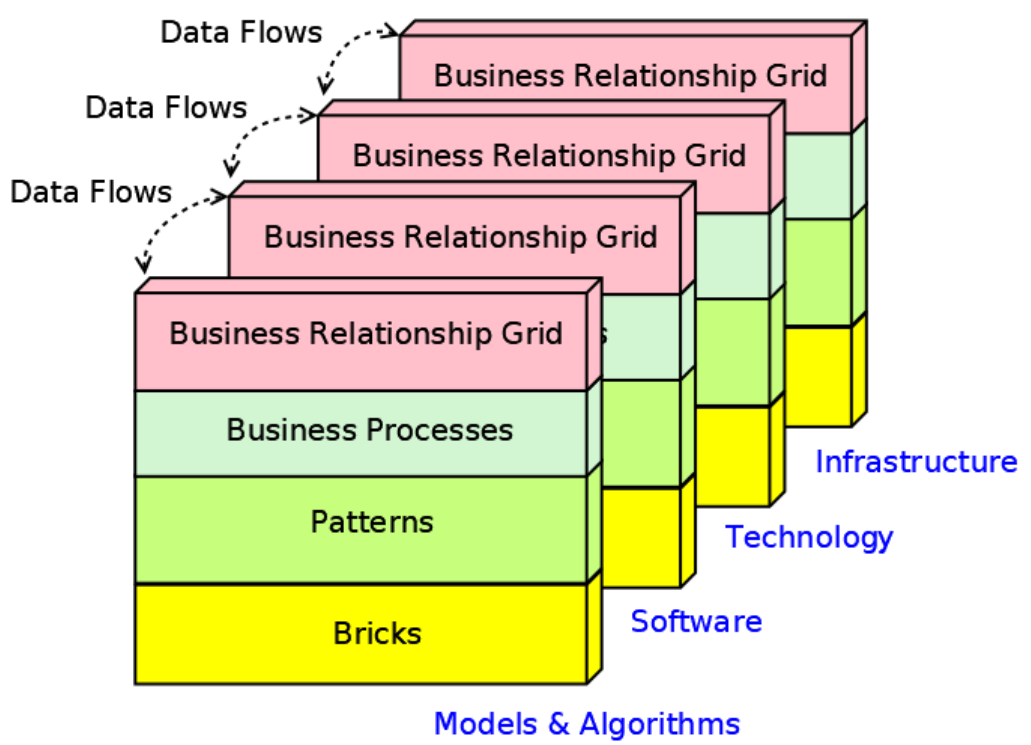

Figure 2. Gartner enterprise architecture.

The functioning of a geographically distributed enterprise is associated with a number of different problems. In particular, a large number of remote units leads to an increase in the complexity of collecting information about their activities and complicates the procedures for processing it. Late receipt of operational information on the state of business processes about construction projects is one of the main factors that reduce the efficiency of management of a construction company, in particular, planning and cost management processes of an enterprise.

To analyze the initial data of a construction project, a significant number of specialists from a construction company are involved, i.e. site managers, supply engineers, designers, cost estimating engineers, etc. However, even with a detailed commercial proposal, a sufficiently high accuracy of assessing the state of business processes is not ensured due to their complexity and impact of various uncertainties.

Usually, production planning carried out only for each local facility. This is due to objective reasons related to the specifics of the construction business. Existing approach does not allow assessing the state of the entire production system of the company, i.e. to optimally distribute its resources across all facilities and make prompt decisions on planning and production management. As a result, the term and cost of the project is determined either by expert or by regulatory methods based on the calculation of the duration of projects' tasks and works, which can cause significant deviations from the actual production targets. 
For the customer of the construction contract, the durations and costs of work are key parameters when choosing a contractor. Penalties for failure to meet contractual deadlines can be up to $30 \%$ of the contract value.

Preliminary planning of production processes in a distributed production system allows coordinating and optimizing the redistribution of resources used between construction sites in the early stages of production. This allows construction manager to develop a consolidated linear-scale schedule (detailed plan of work) for the distribution of the company's own and borrowed resources, based on the plan for resource costs at all construction sites, as well as optimally select the algorithms for synchronizing data flows obtained at various stages of the life cycle of all construction sites.

Improving the efficiency and reliability of assessing the required resources for a local construction site and for the entire set of facilities of a construction enterprise as an organizational and technical system is an urgent task.

This problem is can be solved using network planning methods and BPM technology. It is proposed to solve this problem based on the development of a prototype of the architecture of a construction company within the framework of the Gartner methodology [3].

Consider further the process of developing elements of enterprise architecture for the presented task.

\section{Mathematical models of construction company economic efficiency}

At the stage of developing an information system (see figure 2), basic blocks (models) are needed to solve the problems of optimizing the resources of a construction company. These mathematical models can be used by all participants in production processes and can be part of the enterprise information system [10].

The efficiency of an investment construction project can be estimated using the functions of income $D(t) \rightarrow \max _{t \in S}$ and costs $P(t) \rightarrow \min _{t \in S}$.

In this case, the economic efficiency of the project is defined as follows:

$$
E(t)=D(t)-P(t) \rightarrow \max .
$$

The function of the company's income for a given period of time can be calculated using the formula:

$$
D(t)=\sum_{j=0}^{n} D_{j}(t)
$$

where $j=(1, n), n$ is a number of local construction objects (see figure.1); $D_{j}(t)$ is income from $j$-th local object.

The cost function of a construction company for a given period of time $t$ is represented as follows:

$$
P(t)=\sum_{j=1}^{n} P_{j}\left[t, r_{M_{j}}(t), r_{T_{j}}(t), r_{F_{j}}(t), r_{H_{j}}(t)\right],
$$

where

$r_{M_{j}}(t)$ is the dependence of the costs of material resources of the system for the $j$-th construction object on time;

$r_{T_{j}}(t)$ is dependence of the costs of technical resources of the system for the $j$-th object;

$r_{F_{j}}(t)$ is the dependence of the costs of financial resources of the company for the $j$-th object;

$r_{H_{j}}(t)$ is the dependence of the costs of human resources of the system for the $j$-th object. 
The model also takes into account time constraints $t_{C R_{j}} \leq T_{C_{j}}^{*}$, where $T_{C_{j}}^{*}$ is construction time; $t_{C R_{j}}$ is the duration of the critical path of the $j$-th construction object.

Total company resources constraints $R$ should also be considered:

$$
\begin{aligned}
& \sum_{j=1}^{n} r_{M_{j}}(t) \leq R_{M}(t), \\
& \sum_{j=1}^{n} r_{T_{j}}(t) \leq R_{T}(t), \\
& \sum_{j=1}^{n} r_{F_{j}}(t) \leq R_{F}(t), \\
& \sum_{j=1}^{n} r_{H_{j}}(t) \leq R_{H}(t) .
\end{aligned}
$$

Thus, this statement of the problem can serve as a basis for the development of a pattern of the main goals of a construction company (see figure 2).

Let us further consider the mathematical problem of planning organizational resources for a construction company to create a pattern for planning the resources of a company (see figure 2).

When planning organizational resources, the problem arises of the best distribution of employees (workers) for construction projects and for individual activities (tasks) within the framework of business processes on the constructed objects, taking into account the requirements and restrictions for a given object, for example, with the object's budget and the durations of the works.

The initial data are the following:

- $m$ is number of workers;

- $n$ is number of tasks (activities);

- $d$ is duration of the planned period of the task;

- $W$ is total payroll;

- $K=\left\{k_{1}, k_{2}, \ldots k_{i}, \ldots, k_{m}\right\}$ is set of workers;

- $\quad k_{i}=\left(k_{1}^{i}, k_{2}^{i}\right)$ is vector of characteristics of the $i$-th worker;

- $k_{1}^{i}$ is payment per hour for the $i$-th worker, $k_{2}^{i}$ is assessment of the qualifications of the $i$-th worker, $i=\overline{1, m}$;

- $R=\left\{r_{1}, r_{2}, \ldots r_{\mathrm{j}}, \ldots, r_{n}\right\}$ is set of tasks;

- $r_{j}$ - vector of characteristics of the $j$-th task;

- $r_{j}=\left(r_{1}^{j}, r_{2}^{j}, r_{3}^{j}\right), r_{1}^{j}$ is duration for $j$-th task;

- $r_{2}^{j}$ is assessment of the qualifications required for $j$-th task;

- $r_{3}^{j}$ is the number of days from the beginning of the planned period to the deadline for the completion of the $j$-th task, $j=\overline{1, n}$.

Let the elements of the matrix of feasible assignments $A$ be described as follows:

$$
\begin{aligned}
& a_{i j}= \begin{cases}1, & \text { if } i-\text { th workercan be assigned to } j-\text { th task; } \\
0, & \text { otherwise. }\end{cases} \\
& i=\overline{1, m}, j=\overline{1, n} .
\end{aligned}
$$

Let the matrix of working time be given as $\mathrm{W}=\left\{\mathrm{w}_{i l}\right\}$ where $\mathrm{w}_{i l}$ is the number of free working time for the $i$-th worker on the $l$-th day, $i=\overline{1, m}, \quad l=\overline{1, d}$.

The sought values are the elements of the assignment matrix $\mathrm{X}$ : 


$$
\begin{aligned}
& x_{i j}= \begin{cases}1, & \text { if } i \text { - th workeris assigned to } j \text { - th task; } \\
0, & \text { otherwise. }\end{cases} \\
& i=\overline{1, m}, j=\overline{1, n} .
\end{aligned}
$$

As an objective function, consider the total qualifications of a team of workers:

$$
\sum_{i=1}^{m} \sum_{j=1}^{n} x_{i j} k_{2}^{i} \rightarrow \max
$$

Let us introduce a number of constraints. Only one worker can be assigned to each task: $\sum_{i=1}^{m} x_{i j}=1, \quad j=\overline{1, n}$. Each task can only be assigned to a worker admitted to this task: $\forall x_{i j}=1 \quad a_{i j}=1, i=\overline{1, m}, \quad j=\overline{1, n}$. The qualifications of the appointed worker must be at least equal to the qualifications required to perform the task: $\forall x_{i j}=1 \quad k_{2}^{i} \geq r_{2}^{j}, i=\overline{1, m}, j=\overline{1, n}$. Limitation on the total payroll is $\sum_{i=1}^{m} \sum_{j=1}^{n} x_{i j} k_{1}^{i} r_{1}^{j} \leq \mathrm{W}$.

All the workers must complete the planned task by the specified deadline:

$$
\begin{gathered}
\forall x_{i j}=1 \quad \exists l_{-} \min :\left(1 \leq l_{-} \min \leq d\right) \wedge\left(\min _{S}\left(\sum_{l=l_{-} \min }^{S} w_{i l} \geq r_{1}^{j}\right) \leq r_{3}^{j}\right), \\
i=\overline{1, m}, \quad j=\overline{1, n}
\end{gathered}
$$

where $\min _{S}\left(\sum_{l=l_{-} \min }^{S} w_{i l} \geq r_{1}^{j}\right)$ is the number of days it will take for the $i$-th worker to complete the $j$-th task. Limitation on the maximum workload of workers during the day is $\forall i=\overline{1, m} \sum_{j=1}^{n} x_{i j} r_{1}^{j} \leq \sum_{l=1}^{d} w_{i l}$.

To avoid overloads, underloads in designing a work schedule and tasks without performers, the available organizational are distributed between a set of simultaneously executed construction projects in accordance with the mathematical model of the distribution of organizational resources (5) - (8). The optimal distribution of material, financial and technical resources is calculated in accordance with the mathematical model (1) - (4).

The considered optimization patterns are implemented based on algorithmic patterns to form enterprise architecture within the Gartner framework (see figure 2).

\section{Enterprise architecture patterns design}

During construction, the construction site manager monitors the utilization rate of a particular resource in certain tasks, and uses additional resources if necessary.

This task is solved based on specialized information systems. To implement the network planning algorithm, the main business processes for the construction of a given object are determined, their relationship and duration are determined, a network schedule is drawn up and the total duration of the entire project is determined (see figure 3 ). 


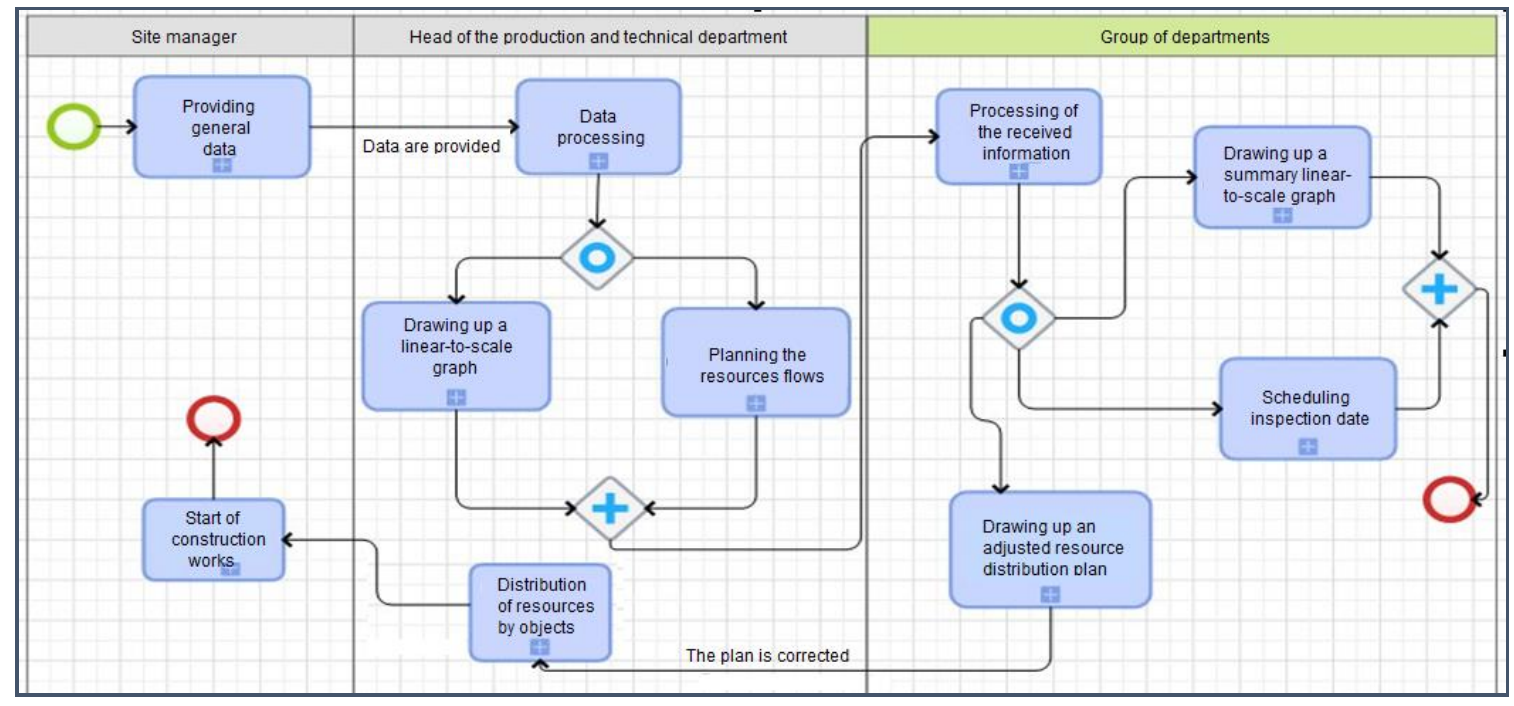

Figure 3. Pattern of business processes.

To solve this problem, a set of models has been implemented using business processes and a visual programming language.

As a means of implementing the models, the BPMN language was chosen, which meets modern requirements and includes a set of effective tools capable of implementing a wide range of tasks. An important point is that a business analyst without the participation of a programmer can perform the design of business processes.

The creation of any business process begins by modelling an organizational structure, on the base of which the executors of processes and sub-processes are determined. Next, a top-level main process is created, in which lower-level sub-processes are included, which provides multi-level detailing.

Based on the developed model of business processes and the corresponding mathematical model, it is possible to find the necessary critical path for the implementation of the sequence of works on the construction of a local construction site.

Algorithms for solving the problem of finding the optimal route on the work execution graph are selected from a set of algorithm patterns $P_{A}$.

Consider further an example of developing an optimal work execution plan, taking into account the given constraints. The considered method of constructing a network plan for a construction site includes four main stages: determining a list of works (see table 1), building a network, calculating and building a time schedule.

Based on the initial data (table 1), a preliminary simplified network schedule for the construction of one of the facilities was developed (see figure 3).

Herewith, critical processes were singled out, on which the total durations for completing construction process depends (for example, the construction of a building frame), and non-critical ones, on which the total durations depend not so rigidly (for example, laying internal communications).

Note that in case a construction company is carrying out similar projects, the schedule (see figure 4) can be used as a design pattern.

This pattern, when filled with data, makes it possible to solve the problem of finding the optimal solution based on the algorithms included in the set of algorithms for finding the optimal path on the graph.

The final cost estimate displays the volume and total cost of work, as well as the size of the wage fund and the total number of standard person-hours. 
Table 1. Initial data for building a network schedule for the construction tasks of a construction object.

\begin{tabular}{clcc}
\hline Designation & \multicolumn{1}{c}{ Process } & Predecessor & Duration (days) \\
\hline A & Site cleaning & - & 1 \\
B & Equipment delivery & - & 2 \\
C & Excavation & A & 1 \\
D & Pouring the foundation & C & 2 \\
E & External plumbing and sewerage works & B, C & 6 \\
F & Building frame construction & D & 10 \\
G & Laying of electrical wiring & F & 3 \\
H & Creating slabs & $\mathrm{G}$ & 1 \\
I & Creating a roof frame & F & 1 \\
J & Internal plumbing and sewerage works & E, H & 5 \\
K & Roofing & I & 2 \\
L & Insulation works & F, J & 1 \\
M & Installation of windows and doors & F & 2 \\
N & Brick lining & L, M & 4 \\
O & Plastering of walls and ceilings & G, J & 2 \\
P & Wall and ceiling cladding & O & 2 \\
Q & Roof insulation & I, P & 1 \\
R & Completion of interior finishing works & P & 7 \\
S & Completion of exterior finishing works & I, N & 7 \\
T & Landscaping & S & 3 \\
\hline
\end{tabular}

Based on the total number of labor costs, the total duration of the work was preliminarily calculated is 48 days, the possible parallel execution of various types of work was also taken into account.

Further, the duration of the critical path was determined on the base of constraints and the model (see figure 4), which decreased to 39 days.

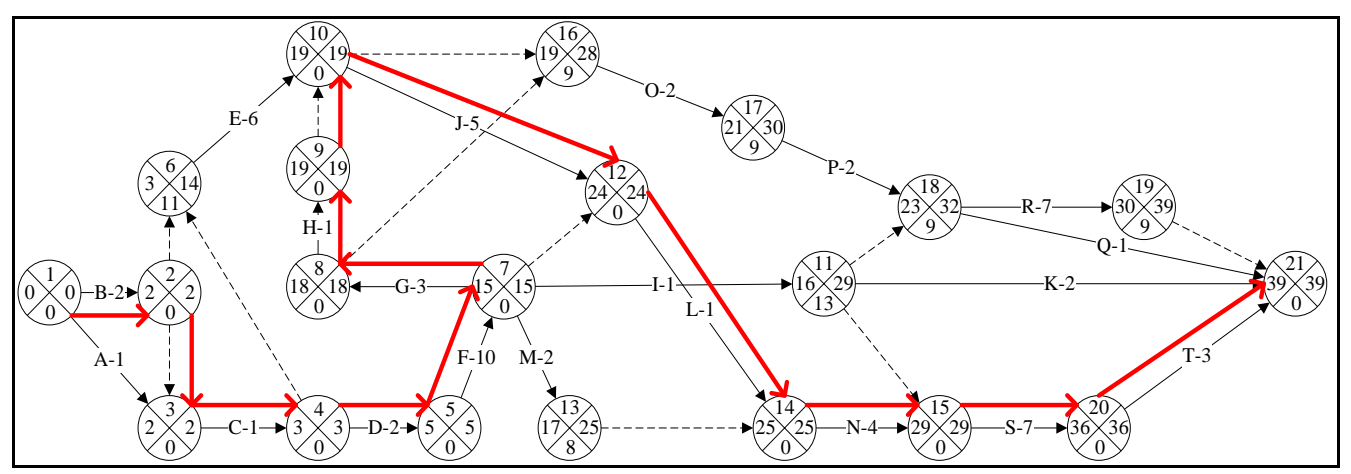

Figure 4. The critical path of construction project.

Thus, we can conclude that the assessment of the timeframe for the completion of all works, based on the proposed approach, turned out to be more accurate. A more accurate forecast of the final terms allows enterprise manager to make a more attractive commercial proposal for the customer, which improves competitive advantage of company.

It also possible to prove that an unreasonable reduction in terms leads to the decline in quality of construction works.

In our case, one of the patterns for shaping the architecture of a construction company can be a typical network schedule for the construction of an object.

On the base of this schedule, it is possible to optimize business processes for each of the construction objects, thereby solving the problem of synchronizing business processes and optimal use of company resources (see figure 5). 


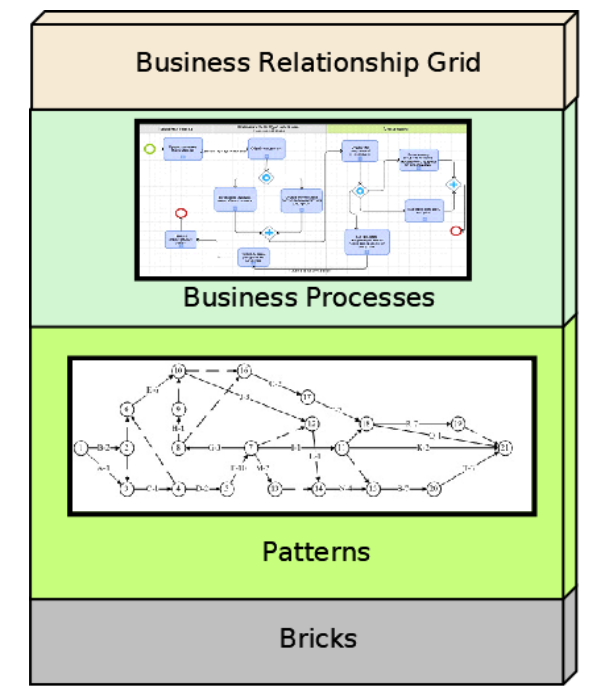

Figure 5. Patterns for Information and Application Layers.

Based on the proposed approach to designing the architecture of an enterprise information system, a prototype of a system was developed for a medium-sized construction company. Typical business processes, a business process optimization model and schedule patterns taking into account the specifics of business processes were used as patterns in the design of the system (see figure 6).

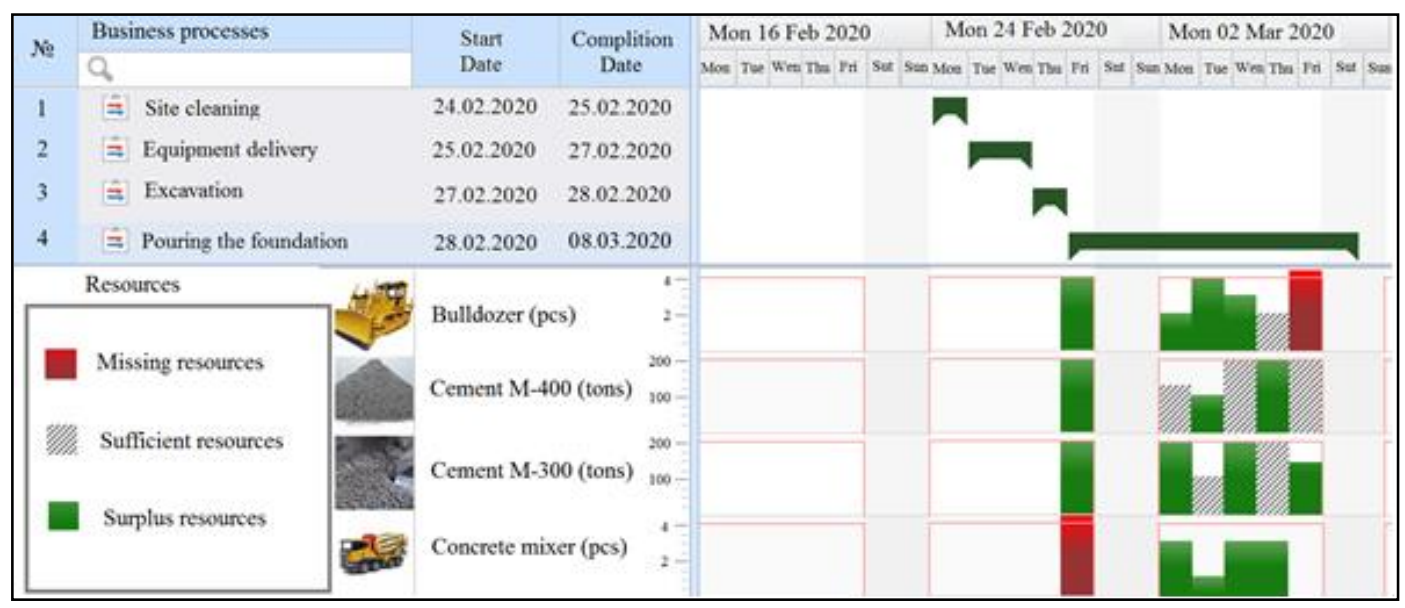

Figure 6. Management module interface of information system.

\section{Conclusions}

Modern construction companies can be attributed to the class of large-scale systems, characterized by a multi-level management system. Usually, companies have a geographically distributed structure and so simultaneously implement business processes at several geographically remote sites.

In order to solve their production tasks, various subcontractors are involved in production and supplies. To improve the efficiency of the construction company, various approaches are used, based on a phased transition from traditional data processing to a digital company within the framework of the Industry 4.0 concepts.

One such approach is the development of Gartner enterprise architecture. Enterprise architecture includes basic elements, patterns, business process models and models of interaction with the external environment.

The article discusses the formulation of the problem associated with the development of the architecture of a construction company as a distributed production system. 
The architectural features of building an information system based on the architecture proposed by Gartner are discussed.

The mathematical formulation of the problem of optimizing business processes is considered. This statement can serve as a basis for the development of the architecture of a construction company. The problem of developing patterns of enterprise architects is considered on the example of a pattern for constructing a schedule for the work of a construction company. A prototype of the architecture of a construction company based on the architecture by Gartner is proposed.

The problem of development of elements of enterprise architecture for a construction company is considered. Mathematical models for optimizing company resources and a typical network schedule for the distribution of work were selected as the main design patterns.

In the development of the information and application layers, field-proven solutions can be used as patterns.

The considered approach to the organization of business processes of a construction company allows solving the problem of optimal scheduling of construction works while simultaneously executing a number of projects by the company and, thus, increasing the management efficiency of a geographically distributed enterprise.

At the same time, it is possible to work in a unified information environment for managers of a construction company, site managers, supply engineers, designers, estimates, etc. It also provides a sufficiently high accuracy in assessing production parameters at the stage of making proposal of construction project, which allows solving the problem of assessing the state of the entire enterprise, optimal allocation of resources among objects and making prompt decisions on planning and production management.

The proposed improvement of planning in construction processes based on business process management technology and the developed mathematical models for planning organizational and technical resources in a distributed construction company makes it possible to increase the efficiency of planning construction processes.

\section{References}

[1] Wilkins J 2017 How Industry 4.0 impacts globalization [Online] Available: https://www.manufacturingglobal.com/technology/how-industry-40-impacts-globalisation [Accessed 06.04.2021]

[2] Davis N 2016 What is the fourth industrial revolution? [Online] Available: https://www.weforum.org/agenda/2016/01/what-is-the-fourth-industrial-revolution [Accessed 06.04.2021]

[3] Enterprise architecture for digital transformation [Online] Available: https://www.gartner.com/en/ [Accessed 10.04.2021]

[4] Bharadwaj A, El Sawy O, Pavlou P and Venkatraman N 2013 Digital business strategy: Toward a next generation of insights MIS $Q 37$ pp 471-482

[5] Hitt L M and Brynjolfsson E 1996 Productivity, business profitability, and consumer surplus: Three different measures of information technology value MIS $Q 20$ pp 121-142

[6] Ross J W, Weill P and Robertson D 2006 Enterprise Architecture as Strategy: Creating a Foundation for Business Execution (Brighton, MA, USA: Harvard Business Press)

[7] Shanks G, Gloet M, Someh I A, Frampton K and Tamm T 2018 Achieving benefits with enterprise architecture J. Strateg. Inf. Syst. 27 pp 139-156

[8] Lee S, Oh S W and Nam K 2016 Transformational and transactional factors for the successful implementation of enterprise architecture in public sector Sustainability $8 \mathrm{p} 456$

[9] Hazen B T, Bradley R V, Bell J E, In J and Byrd T A 2017 Enterprise architecture: A competence-based approach to achieving agility and firm performance Management $193 \mathrm{pp}$ $566-577$

[10] Bottlik G P 2017 An introduction to the mathematics of planning and scheduling (New York: Routledge - Taylor \& Francis) 
[11] Baldwin A and Bordoli D 2014 A handbook for construction planning and scheduling (Oxford: John Wiley \& Sons)

[12] Levy S M 2010 Construction process planning and management: an owner's guide to successful projects (Amsterdam: Elsevier)

[13] Valeev S, Kondratyeva N and Verkhoturov M 2019 Intelligent Decision Support System of Operation Sequencing of Technological Processes Planning Proc. of 2019 International Russian Automation Conference (RusAutoCon) pp 1-6

[14] Tchernykh A, Bychkov I, Feoktistov A, Gorsky S, Sidorov I, Kostromin R, Edelev A, Zorkaltsev V and Avetisyan A 2021 Mitigating Uncertainty in Developing Scientific Applications in Integrated Environment Proc. of the Institute for System Programming of the RAS 33 pp 151-172 